\title{
Proteomic signatures of innate immunity in immunotherapy naiive melanomas correlate with anti-CTLA-4 immunotherapy responsiveness
}

\section{Erin Michelle Taylor}

University of Arkansas for Medical Sciences https://orcid.org/0000-0002-6523-4073

\section{Brian Koss}

University of Arkansas for Medical Sciences

Charity L Washam

University of Arkansas for Medical Sciences

\section{Sara C Shalin}

University of Arkansas for Medical Sciences

Juan C Barreto

University of Arkansas for Medical Sciences

\section{Stefan Graw}

University of Arkansas for Medical Sciences

\section{Stephanie D Byrum}

University of Arkansas for Medical Sciences

\section{Samuel G Mackintosh}

University of Arkansas for Medical Sciences

\section{Rick D Edmondson}

University of Arkansas for Medical Sciences

Alan Tackett ( $\sim$ ajtackett@uams.edu )

\section{Research}

Keywords: CTLA-4, Ipilimumab, immune checkpoint, melanoma, proteomics, innate immunity

Posted Date: September 9th, 2020

DOI: https://doi.org/10.21203/rs.3.rs-69890/v1

License: (c) (i) This work is licensed under a Creative Commons Attribution 4.0 International License.

Read Full License 


\section{Abstract}

Background Factors that determine responsiveness to immune checkpoint inhibitors (ICls) in metastatic melanoma are not completely understood. Failure to respond to ICI therapy can result from primary resistance or the development of secondary resistance to treatment. Understanding the mechanisms that contribute to primary and secondary resistance may help direct which immune checkpoint blockade agents a patient should receive or allow for the development of therapeutics which could be used in combination with immune checkpoint blockade to boost responsiveness.

Methods Formalin-fixed, paraffin embedded, metastatic melanoma tumors were collected from 17 patients prior to receiving anti-CTLA-4 monotherapy. After monotherapy, 5 patients had complete response and 12 had disease progression. The samples were processed for proteomic analysis using filter aided sample preparation and Thermo Tandem Mass Tag (TMT)-labeling of resulting peptides. TMTlabeled samples were fractionated off-line and combined into super fractions. The super fractions were then analyzed by mass spectrometry using an in-line ultra-performance liquid chromatography (UPLC) coupled to a Thermo Orbitrap Fusion Tribrid instrument.

Results Proteomic analysis of metastatic melanoma patient tumors prior to treatment with anti-CTLA-4 therapy identified a total of 4,776 proteins across all patient samples. A random forest model was used to identify the most important proteomic features that could predict responsiveness to treatment. The random forest model had a sensitivity of $80 \%$ and specificity of $67 \%$ for predicting patient response to therapy. A large portion of the proteins elevated in tumors from patients that had complete response to the treatment were associated with innate immunity, particularly pathways involving complement cascade and neutrophil degranulation.

Conclusions Proteomic analysis of immunotherapy naïve metastatic melanoma tissue from patients subsequently treated with anti-CTLA-4 therapy suggests that preexisting innate immune response may be important in responsiveness to treatment. Specifically, elevation of complement cascade or neutrophil degranulation proteins in the tumor microenvironment may increase responsiveness to anti-CTLA-4 monotherapy. Assessing a patient's innate immune status prior to treatment may aid in predicting whether a patient would respond to immune checkpoint inhibition with anti-CTLA-4 monotherapy.

\section{Background}

In recent years, utilizing the immune system to eliminate tumors has become an increasingly popular treatment option in numerous types of cancer. One example of this is the development of immune checkpoint inhibitors (ICls). ICls are monoclonal antibodies that block the interaction of T cell surface receptors, such as PD-1 and CTLA-4, with their respective ligands located on tumor and antigen presenting cells. Both PD-1 and CTLA-4 can act to suppress T cell activity but differ in the mechanism used. CTLA-4 is thought to function by regulating $T$ cell proliferation within lymph nodes during the early stages of an immune response (1). PD-1, however, predominately suppresses T cells located in peripheral 
tissue, which generally happens later in an immune response (1). Immune checkpoint blockade functions by alleviating immune suppression caused by receptor-ligand interaction. ICls are now a standard treatment option for advanced metastatic melanoma patients - with substantially better long-term survival compared to other melanoma treatments. Combination therapy with anti-PD-1 and anti-CTLA-4 antibodies has the highest response rates with $58 \%$ of patients showing at least some degree of response, compared to $45 \%$ or $19 \%$ with either anti-PD- 1 or anti-CTLA-4 monotherapies, respectively (2). However, ICl therapies face numerous problems of partial response and secondary resistance with complete response in only $22 \%$ of patients treated with combination therapy, $19 \%$ treated with anti-PD-1 monotherapy, and $6 \%$ treated with anti-CTLA-4 monotherapy (2). In addition, adverse effects are common among patients treated with immune checkpoint inhibitors. These adverse effects linked to ICls include colitis, pneumonitis, myocarditis and neurotoxic effects and can be sufficiently severe to contribute to death (3). Furthermore, patients treated with combination therapy are more likely to experience an adverse event compared to patients treated with monotherapy (4). While the median duration of response remains unknown in patients treated with anti-PD-1 monotherapy, patients with complete response to anti-CTLA-4 monotherapy tend to have a durable response with a median duration of at least 59 months $(5,6)$. The ability to predict which patients will respond to a monotherapy like anti-CTLA-4 could help define personalized medicine strategies while minimizing the potential for unnecessary adverse effects.

The immune system is known to be a key component in the efficacy of various melanoma treatment options (7). At the genomic level, melanoma is a highly immunogenic tumor with a high frequency of somatic mutations, which frequently corresponds to a greater number of neoantigens produced by the tumor $(8,9)$. High mutational load and neoantigens have been examined in the context of treatment with ICls and have been found to correlate with positive clinical outcome and increased survival (10). While it is known that high mutational load increases immunogenicity of tumors, the complex interactions that occur between the immune system and melanoma are only beginning to be elucidated. Adaptive immunity has been implicated in playing an important role in response to ICls. Melanoma tumors show a greater abundance of $\mathrm{CD}^{+} \mathrm{T}$ cells at the tumor invasive margin in patients that respond to ICI therapy $(11,12)$. Comparison of transcriptomes from pretreatment and on-treatment tumor samples revealed that patients that responded to ICl therapy had a more pronounced increase in $\mathrm{CD} 8^{+} \mathrm{T}$ cells, $\mathrm{CD} 4^{+} \mathrm{T}$ cells, and NK cells, as well as a greater increase in genes associated with lymphocyte activation, immune checkpoints, and cytokine signaling $(13,14)$. At the protein level, lipid metabolism has been found to correspond to increased immunogenicity through elevated antigen presentation and is increased in patients responding to anti-PD-1 monotherapy (15). An intricate relationship exists between adaptive and innate immunity. Innate immunity is known to be essential to the recruitment and activation of leukocytes within tumors in addition to participating in tumoricidal activity (16). Innate immune pathways have been associated with pre- and on-treatment tumors from patients receiving anti-PD-1 therapy (17). Conversely, it has also been found that monocyte and macrophage chemotactic genes (CCL2, CCL7, CCL8, and CCL13) were associated with tumors from patient who did not respond to treatment with anti-PD-1 (10). However, little is known about the role innate immunity plays in responsiveness to anti-CTLA-4 therapy. 
Proteomics can serve as a useful tool in analyzing clinical samples to elucidate the mechanisms involved in responsiveness to immune checkpoint inhibitors. We have previous used label-free proteomics to quantify protein abundance as well as evaluate histone post-translational modifications in a small set of clinical metastatic melanoma samples from patients treated with ICl therapy (12). In addition, Harel et al. used SILAC-based mass spectrometry to analyze a large cohort of clinical samples from patients that received tumor-infiltrating lymphocyte (TIL)-based immunotherapy or anti-PD-1 monotherapy (15). In the current study, we used filter aided sample preparation (FASP) coupled with Thermo Tandem Mass Tag (TMT)-labeling to examine the proteome of immunotherapy naïve metastatic melanoma tumors from 17 patients prior to receiving anti-CTLA-4 monotherapy. The overall objective for the current study was to identify what preexisting features could be important in determining responsiveness to treatment with anti-CTLA-4 monotherapy. Our results implicate proteomic signatures of innate immunity as predictors of response to anti-CTLA-4 monotherapy.

\section{Methods}

\section{Study Design}

The study consisted of formalin-fixed paraffin-embedded (FFPE) metastatic melanoma samples from 17 patients. All of the patients were diagnosed with stage IV metastatic melanoma and went on to receive first line immunotherapy with anti-CTLA-4 monotherapy. Patient information is summarized in Table 1. Experimental methods used in this study were approved and performed in accordance with the University of Arkansas for Medical Sciences (UAMS) Institution Review Board policies and procedures (study \#204543). All samples used in the study were from deidentified archived human tissue biopsies with a waiver for informed consent.

\section{FFPE Tissue Processing.}

For each tumor, we used one $10 \mu \mathrm{m}$ FFPE slide for proteomic studies. The tumor region on each slide was demarcated by a dermatopathologist. The tissue was deparaffinized by heating the slides to $65^{\circ} \mathrm{C}$ for 4.5 minutes, then placed in xylene for 1 minute, $100 \%$ ethanol for 30 seconds, 95\% ethanol for 15 seconds, and rinsed in deionized water 3 times. The tissue was collected from the demarcated region and placed in lysis buffer (100 mM Tris, $2 \%$ SDS, pH 7.6). Tumor area was measured prior to deparaffinization using ImageJ and used to normalize volume of lysis buffer. The samples were heated for 30 minutes at $95^{\circ} \mathrm{C}$ followed by water bath sonication for 5 minutes on high with a Diagenode Bioruptor. After sonication sample were incubated overnight at $65^{\circ} \mathrm{C}$.

\section{Filter Aided Sample Preparation (FASP) and Tandem Mass Tag (TMT)-labeling.}

Each sample was prepared for digestion using $100 \mu \mathrm{L}$ of the patient FFPE tissue solution. Protein was reduced using TCEP (tris(2-carboxyethyl)phosphine hydrochloride) at a final concentration of $10 \mathrm{mM}$ for 30 minutes at $37^{\circ} \mathrm{C}$. Samples were diluted in 11 times the volume of $8 \mathrm{M}$ urea in $100 \mathrm{mM}$ Tris, pH 8.5 (UA buffer) and protein collected by passage through a FASP filter (30,000 MWCO, Sartorius Vivacon 500 
DNA Concentrator VN01H22). Protein immobilized on the filters was treated with $100 \mu \mathrm{L}$ of $50 \mathrm{mM}$ iodoacetamide and incubated in the dark at room temperature for 20 minutes. Following the incubation, the filters were washed with UA buffer and $50 \mathrm{mM}$ TEAB (tetraethylammonium bicarbonate). Trypsin (1 $\mu \mathrm{g})$ was added to each filter and incubated at $37^{\circ} \mathrm{C}$ overnight. The following day, peptides were collected by centrifugation, and formic acid was added to a final concentration of $0.1 \%$. The samples were desalted using a Sep-Pak (Waters) according to the manufacturer's directions and then lyophilized. Lyophilized peptide samples were re-suspended in $28 \mu \mathrm{L}$ of $100 \mathrm{mM}$ TEAB. A pooled reference sample was created by mixing $3 \mu \mathrm{L}$ of each sample. The TMT labels (TMT 10plex Isobaric Label Reagent Set, 0.8 $\mathrm{mg}$, Thermo Scientific) were prepared, and subsequent steps were completed according the manufacturer's directions. Labeled samples and the pooled reference were combined into 3 respective batches (Table 1).

\section{Off-Line Fractionation and In-Line Liquid Chromatography Mass Spectrometry (LC-MS).}

Each of the TMT-labeled batches were fractionated off-line with basic $\mathrm{pH}$ reverse-phase chromatography into 36 fractions using a $100 \times 1.0 \mathrm{C} 18$ column (Acquity BEH, Waters) and UHPLC system (UltiMate 3000, Thermo) with a 40-minute gradient from 99:1 to 60:40 buffer A:B ratio (buffer A: $0.1 \%$ formic acid, $0.5 \%$ acetonitrile; buffer B: $0.1 \%$ formic acid, $99.9 \%$ acetonitrile). The fractions of each batch were then combined into 12 super fractions using a concatenation scheme $(1+13+25,2+14+$ 26, etc.) (18). The 12 super fractions of each batch were then resolved in-line using reverse phase resin (Jupiter Proteo resin, Phenomenex) in a $200 \times 0.075 \mathrm{~mm}$ column using a UPLC system (nanoAcquity, Waters) coupled to a Thermo Orbitrap Fusion Tribrid mass spectrometer. Peptides were eluted with a 60minute gradient from $97: 3$ to $67: 33$ buffer $A: B$ ratio and ionized by electrospray $(2.15 \mathrm{kV})$ followed by mass spectrometric analysis using multi-notch MS3 parameters(19). MS data were acquired using the Fourier Transformation Mass Spectrometry (FTMS) analysis in top-speed profile mode with a resolution of 240,000 and a range of 375 to $1500 \mathrm{~m} / \mathrm{z}$. Collision-induced dissociation (CID) activation was performed with a normalized collision energy of 35.0. MS/MS data were then acquired using the ion trap analyzer in centroid mode with a range of $400-2000 \mathrm{~m} / \mathrm{z}$. Up to $10 \mathrm{MS} / \mathrm{MS}$ precursors were selected for HCD activation with a normalized collision energy of 65.0 using synchronous precursor selection. MS3 reporter ion data was acquired using the FTMS analysis in profile mode at a resolution of 60,000 and a range of $100-500 \mathrm{~m} / \mathrm{z}$.

\section{Data Analysis.}

Mass Spectrometry. Proteins were identified and MS3 reporter ions were quantified using MaxQuant (Version 1.6.0.16, Max Planck Institute) with a parent ion tolerance of $3 \mathrm{ppm}$, a fragment ion tolerance of $0.5 \mathrm{Da}$, and a reporter ion tolerance of $0.001 \mathrm{Da}$, fixed modifications of carbamidomethyl on cysteine, variable modifications of oxidation on methionine and $\mathrm{N}$-terminal acetylation, and 3 missed cleavages possible with trypsin. The MS3 reporter ion intensities were normalized using the Cyclic Loess normalization function from the limma Bioconductor package (20). 
Random Forest. A random forest was generated using the 4,776 proteins identified across all samples (21). The model was built using Salford Random Forest Predictive Modeler. The parameters for the random forest model were tuned to optimize model fit and specified as Target Type:

Classification/Logistic Binary, Number of trees to build: 100000, N predictors: Exactly 500, Seed: 17395, Testing: Out of bag data used for testing (22). The top 483 (Score $\geq 0.04$ ) most important features were used for further analysis.

Reactome Pathway Analysis. Reactome pathway analysis was used to identify pathways associated with the top 483 most important proteins from the random forest (23). The 281 proteins elevated in samples from complete responders were analyzed separately from the 202 proteins decreased. ReacFoams were generated using Reactome pathway analysis and are based on Voronoi tessellation.

Differential Protein Abundance. Significance was calculated on the top 483 most important protein features identified from the random forest model using the moderated t-test. Proteins were considered differentially abundant with a q-value $<0.05$ and a fold change $\geq 2$. Cluster heatmaps were constructed using hierarchical clustering with a variance minimization (Ward) algorithm to calculate the distances between points. Classification of patients into high, moderate, and low groups was based on innate immune protein abundance. For each innate immune protein, the median abundance was calculated on the normalized intensity. High abundance proteins were proteins with a normalized intensity above the median value. The number of high abundance proteins were summed for each patient and the patients were classified based on the percentage of high abundance proteins (PHAP) into the high (PHAP > $66.7 \%$ ), moderate (PHAP $=33.3 \%-66.7 \%)$, or low (PHAP $<33.3 \%$ ) categories.

\section{Tissue Staining}

Slides were cut from FFPE patient tissue blocks to a thickness of $4 \mu \mathrm{m}$ and processed for IHC. Antigen retrieval was perform using citrate buffer, $\mathrm{pH} 6.0$ (Dako Target Retrieval) with incubation in a decloaking chamber (Biocare) for 20 minutes followed by a 30-minute cool down and rinse in deionized water. Peroxidase block (Dako Peroxidase Block) was applied for 10 minutes and rinsed with TBST (trisbuffered saline with tween 20, Dako). Non-specific binding was blocked by applying protein block (Dako Protein Block) for 10 minutes and rinsed with TBST. Primary antibody against AZU1 (Prestige Antibodies: HPA055851) was applied at a 1:100 dilution (diluted in Dako Antibody Diluent with background reducing components) and incubated at room temperature for 1 hour and rinsed with TBST. Secondary antibody, biotinylated goat anti-rabbit (Vector Laboratories: BA-1000) was applied at a 1:400 dilution (diluted in TBST) and incubated at room temperature for 30 minutes and rinsed with TBST. Immunodetection reagent (Vector Elite ABC Kit, Vector Laboratories) was applied and incubated at room temperature for 30 minutes and rinsed with TBST. DAB chromogen (Dako DAB+) was applied and incubated for 3 minutes and rinsed in running tap water. Slides were counterstained with hematoxylin 2 (Richard-Allan Scientific) for 1 minute and rinsed in running tap water. Slides were mounted with permanent mounting media. IHC results were analyzed by a dermatopathologist, blinded to response status, based on the presence or absence of staining in four regions: within tumor, at the tumor-stroma interface, within areas of tumor 
necrosis, and at the tumor-necrosis interface. A Fisher's exact test was used to test for significance between patients with complete response and those with disease progression for each region.

\section{Results}

Proteomic analysis was performed on immunotherapy naïve metastatic melanoma tumor samples. All samples were processed using filter aided sample preparation (FASP) and split into 3 batches with each batch containing both patients with complete response and disease progression (Table 1) (24). A pooled reference sample was created by mixing an equal amount from each of the samples. It was analyzed with each batch and used for normalization of peptide abundance between batches. Each batch was labeled using TMT 10-plex isobaric mass tag labeling reagent $(19,25)$. The workflow of the experiment is shown in Fig. 1. A total of 6,426 proteins were identified using an FDR (false discovery rate) of $1.0 \%$ with 4,776 of the proteins having at least one peptide identified in all of the samples (Supplemental Table 1).

\section{Table 1. Patient Information and TMT batches.}

\begin{tabular}{lllllll} 
Patient & Batch & Sex & Age & Biopsy Location & BRAF Mutation & Response \\
\hline 4 & 1 & female & 45 & lung & no & CR \\
\hline 13 & 1 & male & 72 & small intestines & yes & DP \\
\hline 14 & 1 & male & 29 & lung & yes & DP \\
\hline 15 & 1 & female & 89 & colon & N/A & DP \\
\hline 23 & 1 & female & 85 & lymph node & no & DP \\
\hline 1 & 2 & male & 72 & lymph node & no & CR \\
\hline 2 & 2 & male & 31 & spinal cord & yes & CR \\
\hline 6 & 2 & female & 81 & retroperitoneal & N/A & CR \\
\hline 9 & 2 & female & 62 & colon & no & DP \\
\hline 11 & 2 & female & 82 & brain & no & DP \\
\hline 18 & 2 & male & 68 & lymph node & yes & DP \\
\hline 7 & 3 & female & 42 & colon & no & CR \\
\hline 12 & 3 & male & 55 & brain & yes & DP \\
\hline 16 & 3 & female & 60 & bladder & no & DP \\
\hline 17 & 3 & female & 68 & skin & no & DP \\
\hline 19 & 3 & male & 71 & lymph node & yes & DP \\
\hline 21 & 3 & female & 78 & lymph node & yes & DP \\
\hline
\end{tabular}


All patients received anti-CTLA-4 monotherapy. Response categories: $\mathrm{CR}=$ complete response and $\mathrm{DP}=$ disease progression.

To investigate differences in response to anti-CTLA-4 monotherapy, a random forest classification model was constructed and used to determine how specific proteins from tumors predict response to treatment. The random forest was built using patient response status as the target and all 4,776 proteins as the input features $(21,26-28)$. The random forest used random subsets of 500 proteins to build individual trees with each node of the tree being split on the best protein from the subset and then averaged the predictions from all the trees generated. Overall model accuracy was $71 \%$ with a sensitivity of $80 \%$ and specificity of $67 \%$. The forest was then used to determine the importance of each individual protein in predicting patient response to treatment. Approximately $89 \%$ of the feature importance variables from the 4,776 input proteins are associated with the top 483 proteins (Fig. 2a). Out of the 483 most important proteins, 281 were elevated in tumors from patients with complete response to treatment and the remaining 202 proteins were decreased (Supplemental Table 1). The top 6 most important proteins were METTL3 (N6-adenosine-methyltransferase catalytic subunit), GPKOW (G-patch domain and KOW motifscontaining protein), RDX (radixin), C5 (Complement C5), C4B (Complement C4-B), and FGA (fibrinogen alpha chain), and demonstrated a high degree of separation between the patients from the two response groups (Fig. 2b,c).

Reactome pathway analysis was used to examine the top 483 most important proteins from the random forest and identify pathways that were associated with increased or decreased protein abundance in tumors from patients with complete response to anti-CTLA-4 monotherapy. Analysis of the 202 proteins decreased in tumors from patients with complete response showed pathways involved in metabolism of RNA and RNA splicing (Supplemental Fig. 1). However, only 10 of the 202 proteins decreased met our criteria for differential abundance (q-value $<0.05$, fold change $\geq 2$ ). Analysis of the 281 proteins elevated in tumors from patients with complete response revealed the top upregulated pathways are part of the innate immune response with 104 of the 281 proteins playing a role in innate immunity (Fig. 3a). Upregulated innate immune pathways include antimicrobial peptides, toll-like cascades, cytosolic sensors of pathogen-associated DNA, ROS and RNS production in phagocytes, complement cascade, and neutrophil degranulation (Fig. 3b). Of the 281 proteins elevated, 96 were found to be differentially abundant ( $q$-value $<0.05$, fold change $\geq 2$ ) and 60 of those are part of the innate immune pathways (Fig. 3c).

To determine whether collective abundance of the identified 60 significantly elevated innate immunity proteins correlated with patient responsiveness, protein levels were used to classify patients into high, moderate, or low abundance groups. Patients were classified based on the percentage of high abundance proteins (PHAP): high level (PHAP > 66.7\%), moderate level (PHAP $=33.3 \%-66.7 \%)$, or low level $(\mathrm{PHAP}<$ $33.3 \%)$. Four of five patients with complete response to treatment had high abundance of innate immune proteins and 1 patient had moderate abundance, whereas only 1 out of 12 patients with disease progression had a high abundance of innate immune proteins and the remaining 11 patients had either moderate or low abundance (Fig. 4a). The majority of these 60 significantly elevated innate immunity 
proteins identified from tumors of patients responding to anti-CTLA-4 therapy belong to the complement cascade or neutrophil degranulation pathways (Fig. $\mathbf{4 b , c}$ ).

To confirm proteomics results and examine differences in neutrophil abundance, we used immunohistochemistry (IHC) staining against AZU1 to look for neutrophil presence at four different locations: within the tumor, at the tumor-stroma interface, within necrotic areas, and at the tumor-necrosis interface. AZU1 was selected because it showed the greatest degree of difference between response groups in our proteomics results. High levels of AZU1 staining is indicative of neutrophil presence because it is expressed in neutrophil azurophil granules. AZU1 staining showed significant neutrophil presence at the tumor-necrosis interface in tumors from patients responding to anti-CTLA-4 monotherapy (Fig. 4d).

\section{Discussion}

Metastatic melanoma remains a devastating disease with poor long-term survival for patients who do not respond to treatment with ICls or who develop secondary resistance. Activation of the immune system has been found to play an important role in driving response to various melanoma treatment options including radiation and chemotherapy (7). The immune system can be broken into two major subsystems, the innate immune system and the adaptive immune system. The innate immune system has a cellular component comprised of neutrophils, basophils, eosinophils, macrophages, dendritic cells, mast cells, and natural killer cells as well as an inflammatory component and the complement system. Innate immunity provides an immediate, non-specific response to threats such as pathogens or cells presenting as non-self. The adaptive immune system, on the other hand, generally provides a delayed but specific response to new threats and is comprised of $B$ and $T$ cells, which can develop long-term memory to previously encountered pathogens. Localization of $T$ cells to melanoma tumors prior to immune checkpoint blockade has been found to associate with therapy response, as such identification of markers predicting $T$ cell recruitment to the tumor microenvironment could help identify patients more likely to response $(11,12)$.

In this study, we used TMT quantitative proteomics to investigate metastatic melanoma patient response to anti-CTLA-4 monotherapy (Fig. 1). Proteomic analysis was performed on 17 tumors samples removed prior to patients receiving anti-CTLA-4 immunotherapy (Fig. 2). After monotherapy, 5 patients had a complete response and 12 patients went on to develop disease progression (Table 1). For this sample set, we identified the most important pre-monotherapy proteomic features in determining response. To analyze proteomic data, we used a random forest classification, which is a machine learning technique that is designed to handle high dimensional data and small datasets $(21,26-28)$. The random forest model was generated using all 4,776 proteins identified in all patient samples. Model accuracy was $71 \%$ with a sensitivity of $80 \%$ and specificity of $67 \%$. The top 483 most important proteins accounted for $89 \%$ of differences between response groups. Reactome, a peer-reviewed pathway database, was used to examine the top most important proteins identified from the random forest model $(23,29-31)$. Pathway analysis of the proteins upregulated in metastatic melanoma tumors from patients with complete 
response to treatment showed a high abundance of innate immune proteins indicating a robust innate immune response may be a signature of responding tumors (Fig. 3). Proteins involved in innate immunity accounted for $63 \%$ of the differentially abundant proteins elevated in tumors from responding patients. Neutrophil degranulation and complement cascade were the top two pathways identified with a direct immune function - with $88 \%$ of differentially abundant innate immunity proteins being associated with one of the two pathways (Fig. 4). Both complement cascade and neutrophils play an important role in the innate immune response. The complement cascade is comprised of numerous proteins which undergo proteolytic cascade and play a critical role in the generation of an inflammatory response and phagocytosis via opsonization (32). The complement cascade is part of the innate immune response, but also serves as a bridge between innate and adaptive immunity $(32,33)$. In T cell mediated immunity, the complement cascade can enhance $T$ cell proliferation and decrease apoptosis (33). Activated neutrophils also play an important role in inflammation and secrete pro-inflammatory cytokines (34). Primed neutrophils are also able to activate T cells by presentation of antigens via MHC Class II molecules (34).

Multiple scenarios could potentially explain the reason markers of innate immunity are elevated in tumors from anti-CTLA-4 treated and responding patients. One is that innate immune pathways could be playing a direct role in priming the adaptive immune response to eliminate tumor cells. Patient tumors showing low innate immune response could potentially have more difficulty initiating an adaptive immune response. Alternatively, innate immune markers could be a byproduct of an acute immune response to the tumor in which case the cancer has already been recognized by the immune system. A systematic look at innate immune response before and during anti-CTLA-4 monotherapy may help in developing treatments to improve response to ICls. Previous studies on response to ICI therapy have observed that high mutational load and increased neoantigens are associated with improved clinical outcome and increased survival (10). In addition, increased abundance of $\mathrm{CD} 8^{+} \mathrm{T}$ cells prior to $\mathrm{ICl}$ treatment at the tumor invasive margin have been observed in patients responding to treatment $(11,12)$. High mutational load and neoantigens may increase the likelihood that the melanoma tumor is being recognized by the innate immune system as a problem to be eliminated and triggering a systemic response to the tumor. Furthermore, innate immunity may be driving the recruitment of $\mathrm{CD} 8^{+} \mathrm{T}$ cells to the melanoma prior to the treatment with ICls through the ability of neutrophils to recruit and activate cytotoxic lymphocytes. Future work with a larger cohort of patients will be needed to determine if innate immunity has similar ability to predict response in patients treated with anti-CTLA-4/anti-PD-1 combination therapy or anti-PD-1 monotherapy.

\section{Conclusion}

Proteomic analysis of metastatic melanomas from patients showing complete response to anti-CLTA-4 monotherapy verses disease progression suggests that preexisting innate immune response may be important in determining responsiveness to immune checkpoint inhibitors. In addition, elevated complement cascade or neutrophil degranulation markers in the tumor microenvironment could serve as potential biomarkers to determine whether a person is likely to respond to anti-CTLA-4 monotherapy. 
Improving responsiveness to anti-CTLA-4 monotherapy could lead to a greater number of patients being able to achieve a durable response to treatment while minimizing unnecessary exposures to a drug with a high rate of dangerous adverse effects $(4,6)$. Furthermore, enhanced responsiveness to treatment with anti-CTLA-4 could increase the likelihood of complete response in patients treated with combination therapy. Studies often focus on aspects of the adaptive immune response, such as $\mathrm{CD}^{+}, \mathrm{CD}^{+}$, and regulatory $T$ cells when trying to determine or improve responsiveness to ICls. In contrast, few studies examine the effects of innate immunity on response to checkpoint blockade. This study provides support for the concept that activation of the innate immune system may be a necessary first step in eliciting an adaptive immune response and immune clearance of tumor cells and is worthy of further continued investigation.

\section{Abbreviations}

$\mathrm{ICl}(\mathrm{s})$

immune checkpoint inhibitor(s); TMT:tandem mass tag; UPLC:ultra-performance liquid chromatography; TIL:tumor-infiltrating lymphocyte; FASP:filter aided sample preparation; FFPE:formalin-fixed paraffinembedded; FTMS:Fourier transformation mass spectrometry; CID:collision-induced dissociation; PHAP:percentage of high abundance proteins; FDR:false discovery rate; ROS:reactive oxygen species, RNS:reactive nitrogen species; IHC:immunohistochemistry

\section{Declarations}

\section{Competing interests}

The authors declare that they have no competing interests.

\section{Availability of data and materials}

The datasets generated during the current study are available in the Proteomics Identification Database (PRIDE) repository.

\section{Consent for publication}

Not applicable.

\section{Ethics approval and consent to participate}

The study (study \#204543) was approved by the Institutional Review Board at the University of Arkansas for Medical Sciences (UAMS). All samples used in the study were from deidentified archived tissue biopsies with a waiver for informed consent. 


\section{Funding}

The study was funded by the National Institute of Health (NIH) under grant 1R01CA236209-01. The NIH had no role in the design of the study, collection, analysis, or interpretation of the data, or in writing the manuscript.

\section{Authors' contributions}

EMT and AJT conceived and designed the study. JCB aided in the procurement of patient tissue. EMT prepared the samples. SGM and RDE performed the mass spectrometry. EMT, BK, CLW, SCS, SG, and SDB analyzed and interpreted the data. EMT prepared the figures and tables. EMT and AJT wrote the manuscript. All authors read and approved the final manuscript.

\section{Acknowledgements}

The authors would like to thank the UAMS Proteomics Core and the UAMS Experimental Pathology Core.

\section{References}

1. Buchbinder El, Desai A. CTLA-4 and PD-1 pathways similarities, differences, and implications of their inhibition. Vol. 39, American Journal of Clinical Oncology: Cancer Clinical Trials. Lippincott Williams and Wilkins; 2016. p. 98-106.

2. Larkin J, Chiarion-Sileni V, Gonzalez R, Grob JJ, Rutkowski P, Lao CD, et al. Five-year survival with combined nivolumab and ipilimumab in advanced melanoma. N Engl J Med Massachussetts Medical Society. 2019;381(16):1535-46.

3. Bajwa R, Cheema A, Khan T, Amirpour A, Paul A, Chaughtai S, et al. Adverse Effects of Immune Checkpoint Inhibitors (Programmed Death-1 Inhibitors and Cytotoxic T-Lymphocyte-Associated Protein-4 Inhibitors): Results of a Retrospective Study. J Clin Med Res. Elmer Press, Inc.; 2019;11(4):225-36.

4. Larkin J, Chiarion-Sileni V, Gonzalez R, Grob JJ, Cowey CL, Lao CD, et al. Combined Nivolumab and Ipilimumab or Monotherapy in Untreated Melanoma. N Engl J Med. 2015;373(1):23-34.

5. Hamid O, Robert C, Daud A, Hodi FS, Hwu WJ, Kefford R, et al. Five-year survival outcomes for patients with advanced melanoma treated with pembrolizumab in KEYNOTE-001. Ann Oncol Elsevier Masson SAS. 2019;30(4):582-8.

6. Schadendorf D, Hodi FS, Robert C, Weber JS, Margolin K, Hamid O, et al. Pooled analysis of long-term survival data from phase II and phase III trials of ipilimumab in unresectable or metastatic melanoma. J Clin Oncol American Society of Clinical Oncology. 2015;33(17):1889-94.

7. Tiffen JC, Gallagher SJ, Tseng H-Y, Filipp FV, Fazekas de St. Groth B, Hersey P. EZH2 as a mediator of treatment resistance in melanoma. Pigment Cell Melanoma Res NIH Public Access. 2016;29(5):500- 
7.

8. Passarelli A, Mannavola F, Stucci LS, Tucci M, Silvestris F. Immune system and melanoma biology: A balance between immunosurveillance and immune escape. Vol. 8, Oncotarget. Impact Journals, LLC; 2017. p. 106132-42.

9. Lawrence MS, Stojanov P, Polak P, Kryukov GV, Cibulskis K, Sivachenko A, et al. Mutational heterogeneity in cancer and the search for new cancer-associated genes. Nature. 2013;499(7457):214-8.

10. Hugo W, Zaretsky JM, Sun L, Song C, Moreno BH, Hu-Lieskovan S, et al. Genomic and Transcriptomic Features of Response to Anti-PD-1 Therapy in Metastatic Melanoma. Cell. 2016;165(1):35-44.

11. Tumeh PC, Harview CL, Yearley JH, Shintaku IP, Taylor EJM, Robert L, et al. PD-1 blockade induces responses by inhibiting adaptive immune resistance. Nature NIH Public Access. 2014;515(7528):568-71.

12. Shields BD, Mahmoud F, Taylor EM, Byrum SD, Sengupta D, Koss B, et al. Indicators of responsiveness to immune checkpoint inhibitors. Sci Rep Springer US. 2017;7(1):807.

13. Riaz N, Havel JJ, Makarov V, Desrichard A, Urba WJ, Sims JS, et al. Tumor and Microenvironment Evolution during Immunotherapy with Nivolumab. Cell. 2017;171(4):934-49.e15.

14. Chen P-L, Roh W, Reuben A, Cooper ZA, Spencer CN, Prieto PA, et al. Analysis of Immune Signatures in Longitudinal Tumor Samples Yields Insight into Biomarkers of Response and Mechanisms of Resistance to Immune Checkpoint Blockade. Cancer Discov NIH Public Access. 2016;6(8):827-37.

15. Harel M, Ortenberg R, Karthik S, Kaech SM, Markel G, Geiger T. Proteomics of Melanoma Response to Immunotherapy Reveals Mitochondrial Dependence. Cell. 2019.

16. Moynihan KD, Irvine DJ. Roles for Innate Immunity in Combination Immunotherapies. Cancer Res. 2017;77(19).

17. Auslander N, Zhang G, Lee JS, Frederick DT, Miao B, Moll T, et al. Robust prediction of response to immune checkpoint blockade therapy in metastatic melanoma. Nat Med Springer US. 2018;24(10):1545-9.

18. Wang Y, Yang F, Gritsenko MA, Wang Y, Clauss T, Liu T, et al. Reversed-phase chromatography with multiple fraction concatenation strategy for proteome profiling of human MCF10A cells. Proteomics. NIH Public Access. 2011;11(10):2019-26.

19. McAlister GC, Nusinow DP, Jedrychowski MP, Wühr M, Huttlin EL, Erickson BK, et al. MultiNotch MS3 enables accurate, sensitive, and multiplexed detection of differential expression across cancer cell line proteomes. Anal Chem American Chemical Society. 2014;86(14):7150-8.

20. Ritchie ME, Phipson B, Wu D, Hu Y, Law CW, Shi W, et al. limma powers differential expression analyses for RNA-sequencing and microarray studies. Nucleic Acids Res. 2015;43(7):e47.

21. Breiman L. Random forests. Mach Learn Springer. 2001;45(1):5-32.

22. Scornet E. Tuning parameters in random forests. ESAIM Proc Surv. 2017;60:144-62. 
23. Jassal B, Matthews L, Viteri G, Gong C, Lorente P, Fabregat A, et al. The reactome pathway knowledgebase. Nucleic Acids Res. Oxford University Press; 2020;48(D1):D498-503.

24. Erde J, Loo RRO, Loo JA. Enhanced FASP (eFASP) to increase proteome coverage and sample recovery for quantitative proteomic experiments. J Proteome Res American Chemical Society. 2014;13(4):1885-95.

25. Maes E, Valkenborg D, Mertens I, Broeckx V, Baggerman G, Sagaert X, et al. Proteomic analysis of formalin-fixed paraffin-embedded colorectal cancer tissue using Tandem Mass Tag protein labeling.

26. Jagga Z, Gupta D. Machine learning for biomarker identification in cancer research developments toward its clinical application. Vol. 12, Personalized Medicine. Future Medicine Ltd.; 2015. p. 371-87.

27. Jagga Z, Gupta D. Classification models for clear cell renal carcinoma stage progression, based on tumor RNAseq expression trained supervised machine learning algorithms. In: BMC Proceedings. BioMed Central Ltd.; 2014. p. S2.

28. Verikas A, Gelzinis A, Bacauskiene M. Mining data with random forests: A survey and results of new tests. Pattern Recognit. Pergamon; 2011 Feb 1;44(2):330-49.

29. Fabregat A, Sidiropoulos K, Viteri G, Forner O, Marin-Garcia P, Arnau V, et al. Reactome pathway analysis: A high-performance in-memory approach. BMC Bioinformatics BioMed Central Ltd. 2017;18(1):142.

30. Marco-Ramell A, Palau-Rodriguez M, Alay A, Tulipani S, Urpi-Sarda M, Sanchez-Pla A, et al. Evaluation and comparison of bioinformatic tools for the enrichment analysis of metabolomics data. BMC Bioinformatics BioMed Central Ltd. 2018;19(1):1-11.

31. Wu X, Hasan M, Al, Chen JY. Pathway and network analysis in proteomics. J Theor Biol Academic Press. 2014;362:44-52.

32. Dunkelberger JR, Song WC. Complement and its role in innate and adaptive immune responses. Cell Res Nature Publishing Group. 2010 Jan;15(1):34-50. 20 (.

33. Kwan WH, Van Der Touw W, Heeger PS. Complement regulation of T cell immunity. Immunol Res NIH Public Access. 2012 Dec;54(1-3):247-53.

34. Wright HL, Moots RJ, Bucknall RC, Edwards SW. Neutrophil function in inflammation and inflammatory diseases. Rheumatology. 2010;49(9):1618-31.

\section{Figures}




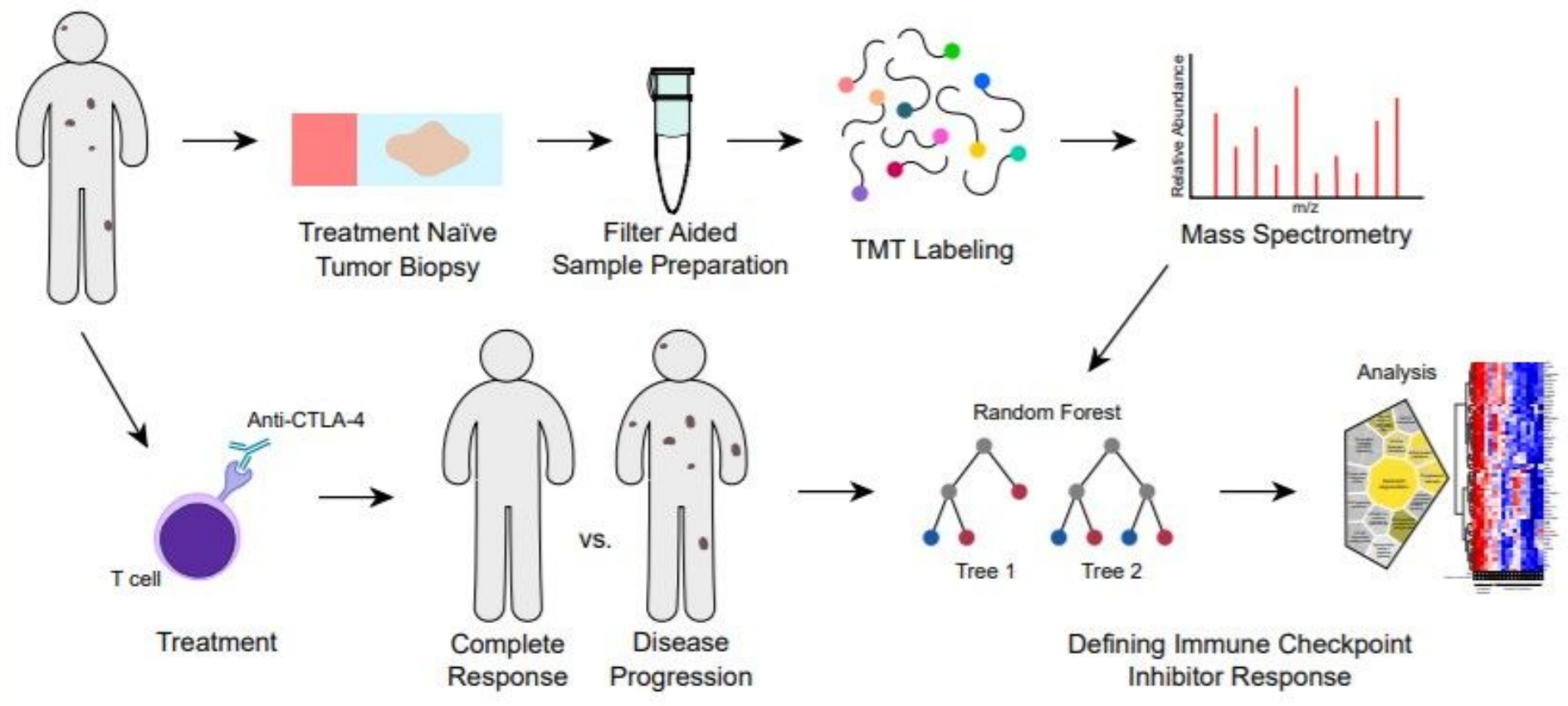

Figure 1

Proteomics workflow. 


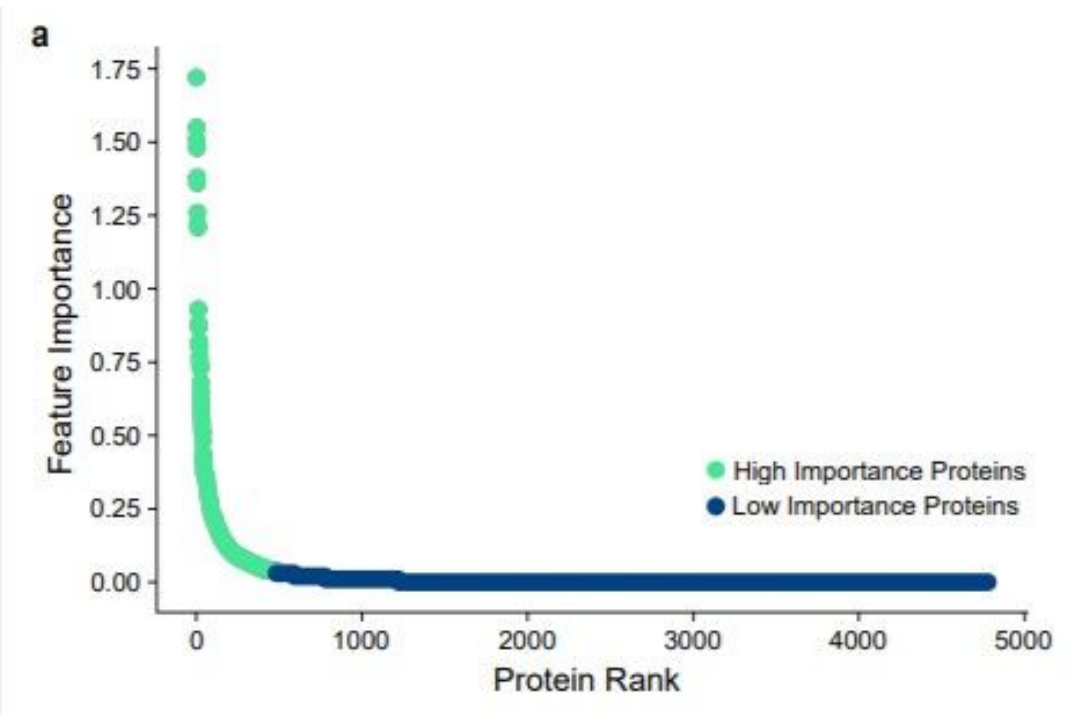

b
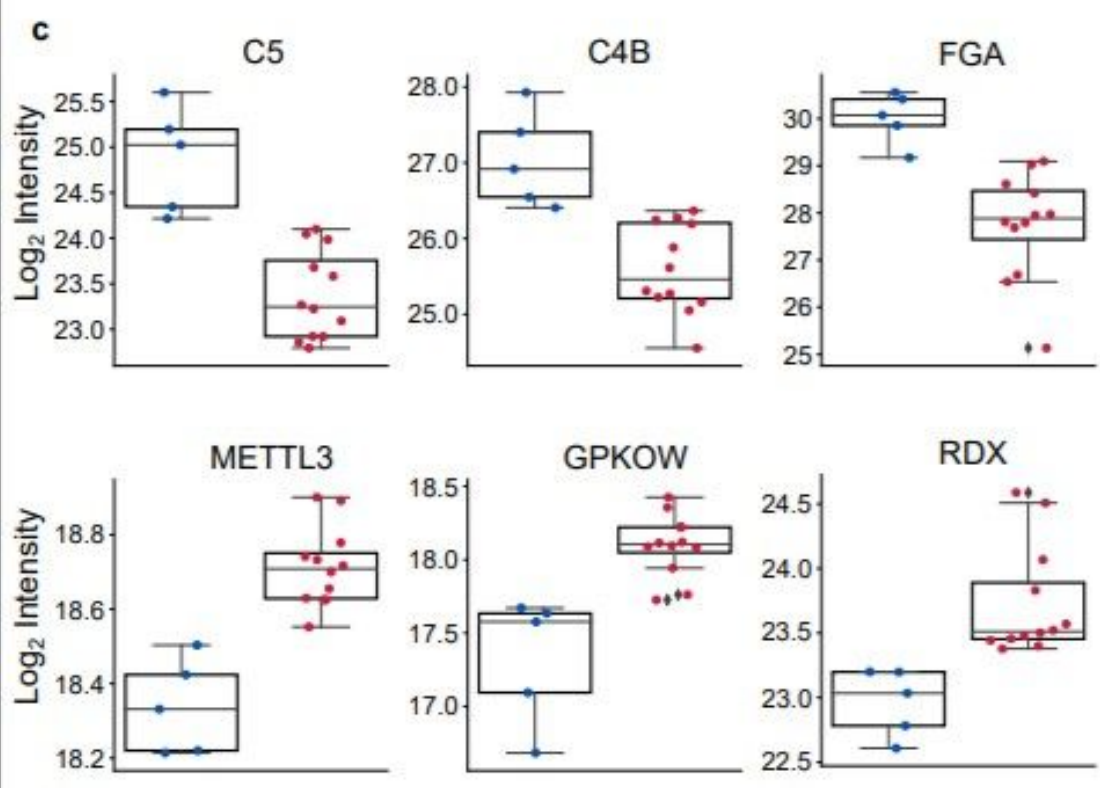

- Complete Response Disease Progression

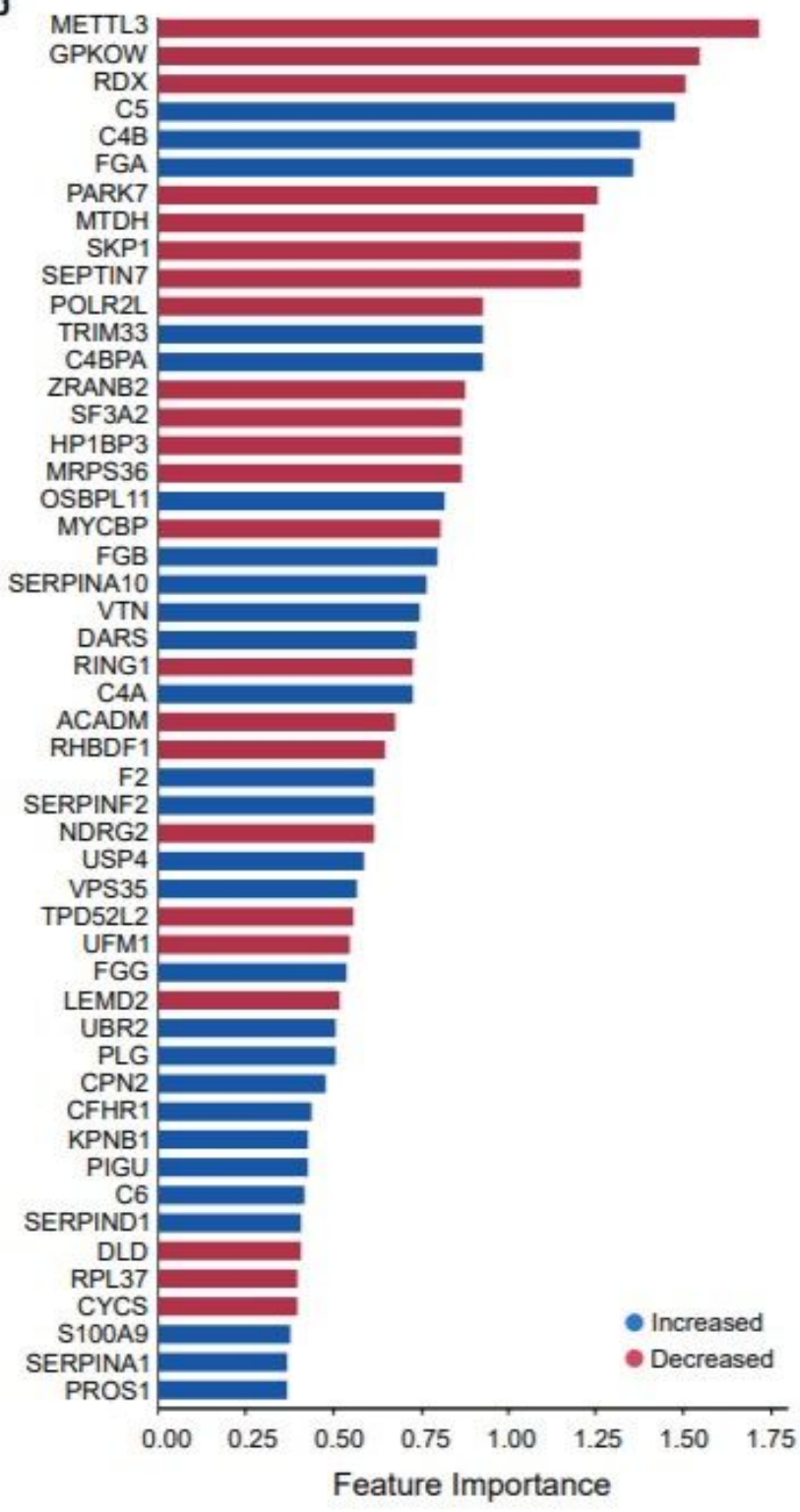

\section{Figure 2}

Random forest analysis shows proteins most important in predicting patient response to anti-CTLA-4 monotherapy. a Scatter plot of all 4,776 proteins ordered according to rank of feature importance. $b$ Top 50 most important proteins in predicting patient response. c Boxplots showing distribution of patients for each of the top 6 most important proteins. 


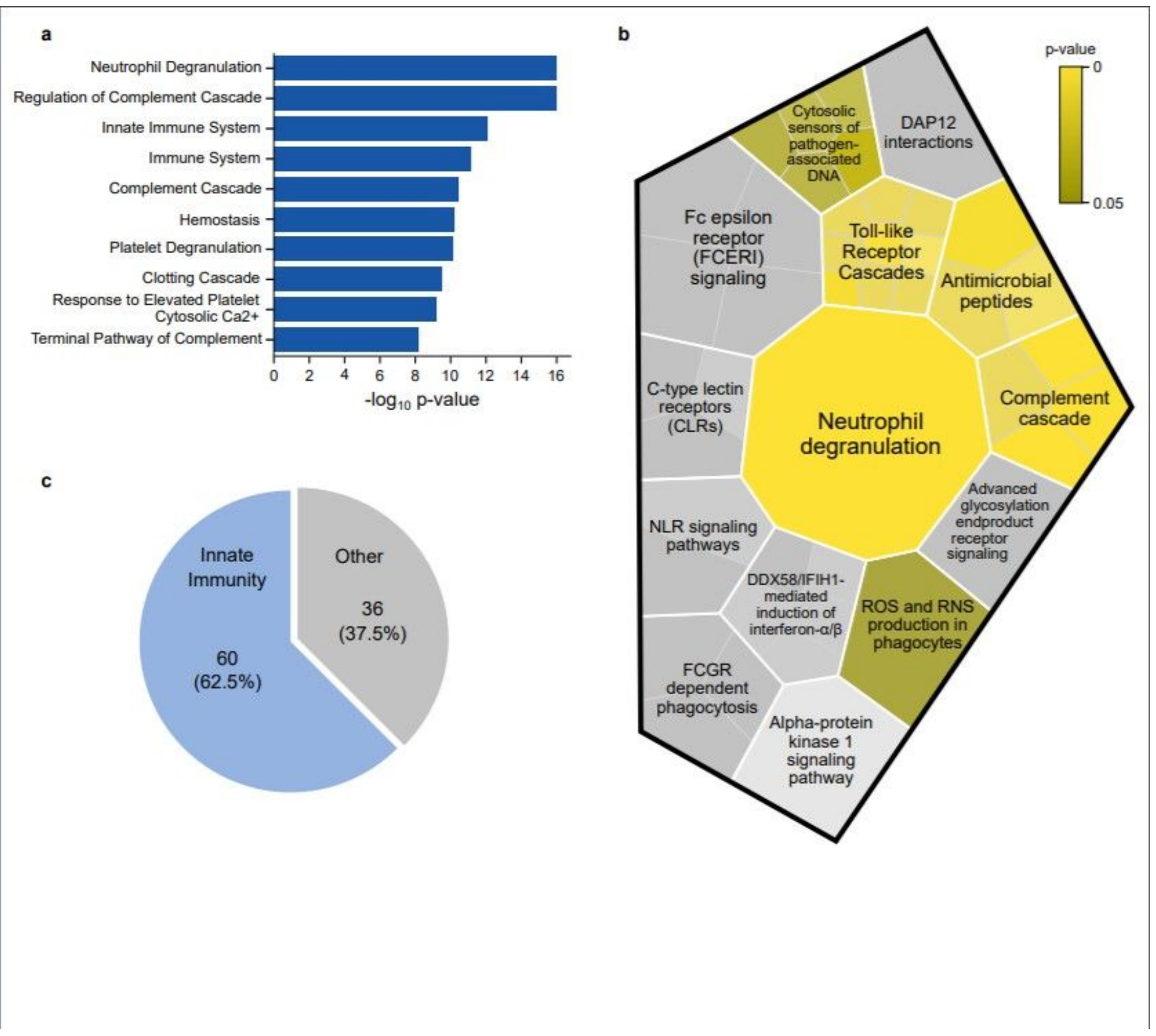

\section{Figure 3}

Upregulated pathways in tumors from patients with complete response are associated with innate immunity. a The top 10 pathways identified from Reactome pathway analysis in metastatic melanoma tumors from patients with complete response. $b$ ReacFoam analysis showing the most substantially increased pathways associated with innate immunity. c Pie chart of all differentially abundant proteins elevated in tumors from patients with complete response (q-value $<0.05$, fold change $\geq 2$ ). 


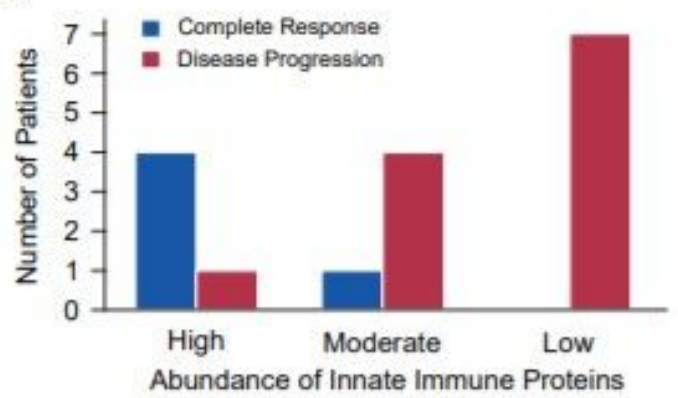

b

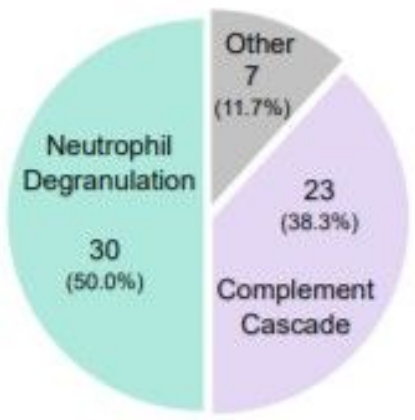

d
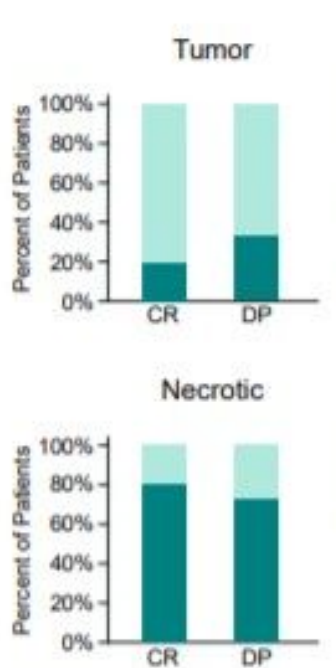

$C R=$ Complete Response
Tumor-Stroma Interface

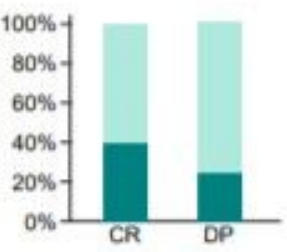

Tumor-Necrosis

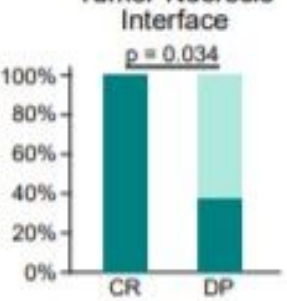

$\mathrm{DP}=$ Disease Progression

Neutrophils by AZU1 Staining

- Present Absent

c

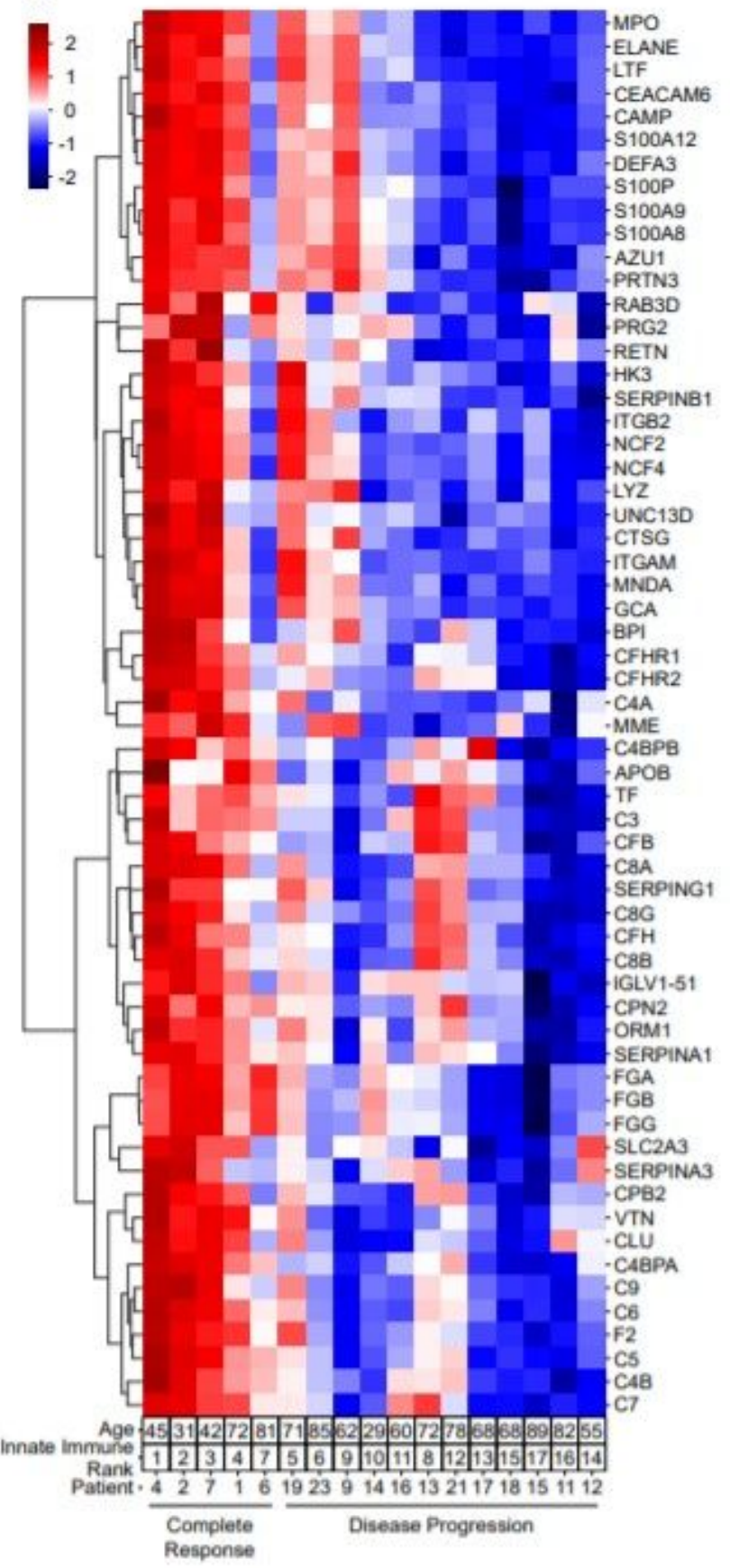

Figure 4

Complete response tumors show increased abundance of proteins related to complement cascade and neutrophil degranulation. a Patient classified into high, moderate, or low innate immunity protein abundance by proteomic analysis. b Pie chart showing the ratio of innate immune proteins associated with neutrophil degranulation and complement cascade. c Clustered heatmap of the 60 differentially 
abundant innate immune proteins. $d$ Presence of neutrophils using immunohistochemistry staining for AZU1 shown by tumor location and grouped by patient response.

\section{Supplementary Files}

This is a list of supplementary files associated with this preprint. Click to download.

- SupplementalTable1.xIsx

- SupplementalFigure1.pdf 